\title{
CARACTERIZAÇÃO DA MATÉRIA ORGÂNICA PARTICULADA DOS ÚLTIMOS 10 MIL ANOS A PARTIR DE UM TESTEMUNHO DO PARQUE ESTADUAL DO RIO DOCE, MG, BRASIL: IMPLICAÇÕES PALEOAMBIENTAIS
}

\author{
FERNANDA MARA FONSECA-SILVA \\ Programa de Pós-Graduação em Evolução Crustal \& Recursos Naturais, Departamento de Engenharia Geológica, \\ UFOP, Morro do Cruzeiro, s/n, 35400-000, Ouro Preto, MG, Brasil.fernandamarafonseca@gmail.com \\ MARCELO DE ARAUJO CARVALHO \\ Laboratório de Paleoecologia Vegetal, Departamento de Geologia \& Paleontologia, Museu Nacional/UFRJ, \\ Quinta da Boa Vista, s/n, 22040-040, Rio de Janeiro, RJ, Brasil.mcarvalho@mn.ufrj.br \\ SÉRVIO PONTES RIBEIRO \\ Departamento de Biodiversidade, Evolução \& Meio Ambiente, UFOP, Morro do Cruzeiro, s/n, \\ 35400-000, Ouro Preto, MG, Brasil.spribeiro@iceb.ufop.br
}

\begin{abstract}
CHARACTERIZATION OF PARTICULATE ORGANIC MATTER OF THE LAST 10.000 YEARS FROM A CORE OF STATE PARK OF DOCE RIVER, MG, BRAZIL: PALAEOENVIRONMENTAL IMPLICATIONS. This paper presents the results of a palynofacies study from a Holocene core of a Lake (Lc2), State Park of Doce River, MG, Brazil. The analysis of palynofacies was carried out on 17 samples of a core of $90 \mathrm{~cm}$ (Lc2-01), dated at $80 \mathrm{~cm}$ depth in 10,191 cal. years BP, $65 \mathrm{~cm}$ in 9,640 cal. years BP, $35 \mathrm{~cm}$ in 7,905 cal. years BP and $10 \mathrm{~cm} 102$ cal. years BP. The core Lc2-01 shows an increased level of silt/clay upwards, supporting the idea that the depositional process involved low energy. The identification of the organic components yielded three distinct palynofacies. The Palynofacies 1 (Pf1) is composed of cuticles, corresponding to a fluvio-lacustrine environment; Palynofacies 2 (Pf2) dominant elements are phytoclasts (opaque and nonopaque), representing woody components and Palynofacies 3 (Pf3) is composed of a predominance of amorphous organic matter and palynomorphs (spores, pollen grains and freshwater algae). The stratigraphic distribution of the palynofacies, allowed the identification of three phases in core Lc2-01. Phase 1 (10,375-9,351 cal. years BP) has a predominance of Pf2, suggesting a fluvio lacustrine environment of high energy and even at 9,351 cal. years BP the Doce river bed was not closed. From 9,351-7,905 cal. years BP (phase 2) there was a continuing increase in Pf2 and a severe elevation of Pf1, indicating the beginning of the closing of the valleys of the old Doce river bed. The phase (7,905-102 cal. years BP), demonstrates an abrupt rise of $\mathrm{Pf}$, suggesting anoxic-dioxic compatible with shallow lacustrine environment or paludal water.
\end{abstract}

Key words: palynofacies, Holocene, State Park of Doce River, Brazil.

RESUMO - Este trabalho apresenta a caracterização da matéria orgânica particulada de um testemunho de sondagem de 90 cm (Lc2-01), em um lago assoreado, localizado no Parque Estadual do Rio Doce, MG, Brasil. A análise de palinofácies foi realizada em 17 amostras, datado na profundidade de $80 \mathrm{~cm}$ em $10.191 \mathrm{cal}$. anos AP, $65 \mathrm{~cm}$ em $9.640 \mathrm{cal}$. anos AP, $35 \mathrm{~cm}$ em 7.905 cal. anos AP e $10 \mathrm{~cm} 102$ cal. anos AP. O Lc2-01 apresenta uma granodecrescência para o topo, sustentando a ideia de que o processo deposicional no lago envolveu baixa energia. Com base na análise qualitativa da MOP foi possível identificar três associações de palinofácies distintas. A palinofácies 1 (Pf1) é composta basicamente de cutículas, a Palinofácies 2 (Pf2) composta de fitoclastos opacos e não opacos representando os componentes lenhosos e a Palinofácies 3 (Pf3) composta por matéria orgânica amorfa e palinomorfos. A distribuição estratigráfica das associações das palinofácies permitiu dividir a seção estudada em três fases. A Fase 1 (10.375-9.351 cal. anos AP) possui o predomínio da Pf2, sugerindo um ambiente de maior energia, interpretado como flúvio-lacustre, pois até 9.351 cal. anos AP a região não tinha sofrido com o fechamento do leito do rio Doce. Na fase 2 (9.351-7.905 cal. anos AP) observou-se o contínuo aumento na Pf 2 e uma severa elevação da Pf1, apontando o início do fechamento dos vales do antigo leito do rio Doce. A Fase 3 (7.905-102 cal. anos AP) é caracterizada por um abrupto aumento da Pf3, sugerindo condições mais redutoras compatíveis com lâmina d'água de pouca profundidade, interpretada como um ambiente lacustre ou paludal.

Palavras-chave: palinofácies, Holoceno, Parque Estadual do Rio Doce, Brasil. 


\section{INTRODUÇÃO}

O conceito de palinofácies foi introduzido por Combaz (1964) e expandido por Tyson (1995), que proferiu que as palinofácies são grupos de sedimentos contendo uma assembleia distinta de constituintes orgânicos que podem ser reconhecidos por microscopia ou estar associado. Já Traverse (1994) definiu que o termo palinofácies deveria ser utilizado para referir-se à concentração local de certos palinomorfos particulares indicativos de um tipo de biofácies e que a pesquisa de palinofácies voltada para estudos geológicos sobre o paleoambiente de deposição de uma determinada rocha deveria ser denominada de palinolitofácies. Assim, o estudo de palinofácies compreende na análise da fração particulada da matéria orgânica contida em rochas sedimentares, sendo ela dividida em duas frações, $o$ querogênio (matéria orgânica particulada - MOP) e o betume (matéria orgânica extraível) (Forsman \& Hunt, 1958).

O objetivo do estudo de palinofácies é a integração de todos os aspectos da assembleia de componentes orgânicos particulados bem como a identificação dos componentes palinológicos individuais, a classificação dos grupos e subgrupos da MOP e a determinação das proporções relativas atentando particularmente, para tamanho, forma e estado de preservação. Nesse caso, significa ter uma rigorosa subdivisão das categorias, com o objetivo de identificar qualquer variação quantitativa que poderia estar relacionada aos principais controles na distribuição da matéria orgânica e, assim, utilizar esses fatores na determinação do significado paleoambiental (Mendonça Filho et al., 2010).

Dado que as condições sejam favoráveis à preservação de matéria orgânica amorfa (MOA), fitoclastos e palinomorfos em sedimentos provenientes de lagos, turfeiras e pântanos antigos registram então a memória e consequentemente a paleovegetação da região (Bloom, 1978). Há uma grande escassez de estudos sobre a distinção dos parâmetros de palinofácies em depósitos fluviais continentais quaternários dentro do sistema lacustre do Parque Estadual do Rio Doce (PERD), o maior sistema lacustre natural da América do Sul, circundado pelas últimas reservas contínuas bem preservadas de Mata Atlântica de Minas Gerais.

O PERD é um ambiente que vem sofrendo assoreamentos progressivos ao longo do Quaternário (Barbosa \& Kohler, 1981) e evoluindo sob a influência das variações climáticas e neotectônicas (Mello, 1997). Assim, esse trabalho tem como objetivo geral caracterizar a matéria orgânica particulada contida em amostras de um testemunho de sondagem de um lago assoreado no Parque Estadual do Rio Doce, denominado de Lago Lc2, identificando as condições de deposição durante a evolução holocênica do lago, bem como a relação dos eventos ocorridos nessa região. O Lc2 mostrou-se um ambiente propício à coleta de testemunhos sedimentares com a finalidade de reconstituição paleoambiental por estar localizado em um local de difícil acesso, cercado por vegetação nativa, onde a interferência antrópica foi pequena ou inexistente. Até os dias atuais é provável que as informações registradas nestes sedimentos não foram desta forma, adulteradas.

\section{CARACTERIZAÇÃO DA ÁREA DE ESTUDO}

A bacia hidrográfica do rio Doce está localizada na região Sudeste, entre os paralelos $18^{\circ} 45^{\prime}$ e $21^{\circ} 15^{\prime}$ de latitude sul e os meridianos $39^{\circ} 55^{\prime}$ e $43^{\circ} 45^{\prime}$ de longitude oeste, abrangendo uma área de drenagem de $83.400 \mathrm{~km}^{2}$, dos quais $86 \%$ pertencem ao Estado de Minas Gerais e o restante ao Estado do Espírito Santo.

O PERD foi criado em 1944 e é administrado pelo Instituto Estadual de Florestas de Minas Gerais (IEF-MG) desde 1962. Está situado na porção sudoeste do Estado de Minas Gerais, na região do Vale do Aço, sendo limitado ao norte e a leste pelos rios Piracicaba e Doce respectivamente, a oeste pelos municípios de Marliéria e Timóteo e ao sul por Dionísio (Figura 1). O PERD possui 36.970 hectares onde se encontra um sistema composto por aproximadamente 160 corpos lacustres ( $10 \%$ de sua área aproximadamente), sendo cerca de 40 lagos em processos avançados de assoreamento. De acordo com Pflug (1969), a formação desses complexos lacustres seria uma consequência do fechamento dos vales secundários do Rio Doce, desde o fim do Pleistoceno com paleoambientes estáveis. Já Meis (1978), afirmou que a natureza dos sedimentos indica mudanças do paleoambiente e variações nas condições paleohidrológicas durante o Holoceno. Mello (1997) descreveu que a origem do complexo lacustre no PERD está relacionada, principalmente, à neotectônica local, sendo a primeira fase caracterizada pela transcorrência dextral/Leste-Oeste (E-W) e atuou a partir do final do Pleistoceno. Já a segunda fase de movimentações tectônicas ocorreu durante o Holoceno Médio e é caracterizada pelo regime extensional/Noroeste-Sudeste (NW-SE), o qual foi responsável pela ocorrência de falhamentos que provocaram os desnivelamentos e basculamentos nos leitos dos canais tributários do rio Doce, funcionando assim, como barragens dando origem aos sistemas lacustres no PERD.

O curso do rio Doce é intensamente controlado pelas estruturas fundamentais do embasamento pré-cambriano, com sua formação provavelmente vinculada a mecanismos tectônicos rúpteis, de idade cenozoica (Justo, 2003).

\section{MATERIAL E MÉTODOS}

\section{Testemunho}

O Lago Lc2, escolhido para este trabalho, situa-se na região centro-norte do PERD, próximo à Guarita do Salão Dourado. Ele é margeado por vegetação nativa, porém alguns de seus braços a Oeste estão fora do Parque. A sua parte central ainda é lago e os dois braços principais são assoreados. No Lc2 foi coletado o testemunho denominado Lc2-01, localizado pelas coordenadas de $42^{\circ} 34^{\prime} 47^{\prime \prime} S$ e $19^{\circ} 39^{\prime} 01$ 'O (UTM $0753747 \mathrm{~N} ; 7825378$ O). A sondagem foi realizada por percussão utilizando tubos de PVC. Foram selecionadas 17 amostras, ao longo de $90 \mathrm{~cm}$ de profundidade do testemunho para o estudo de palinofácies.

O tratamento químico das amostras foi realizado no Laboratório de Geoquímica Ambiental da Universidade Federal de Ouro Preto (LGqa/DEGEO/UFOP), de acordo 


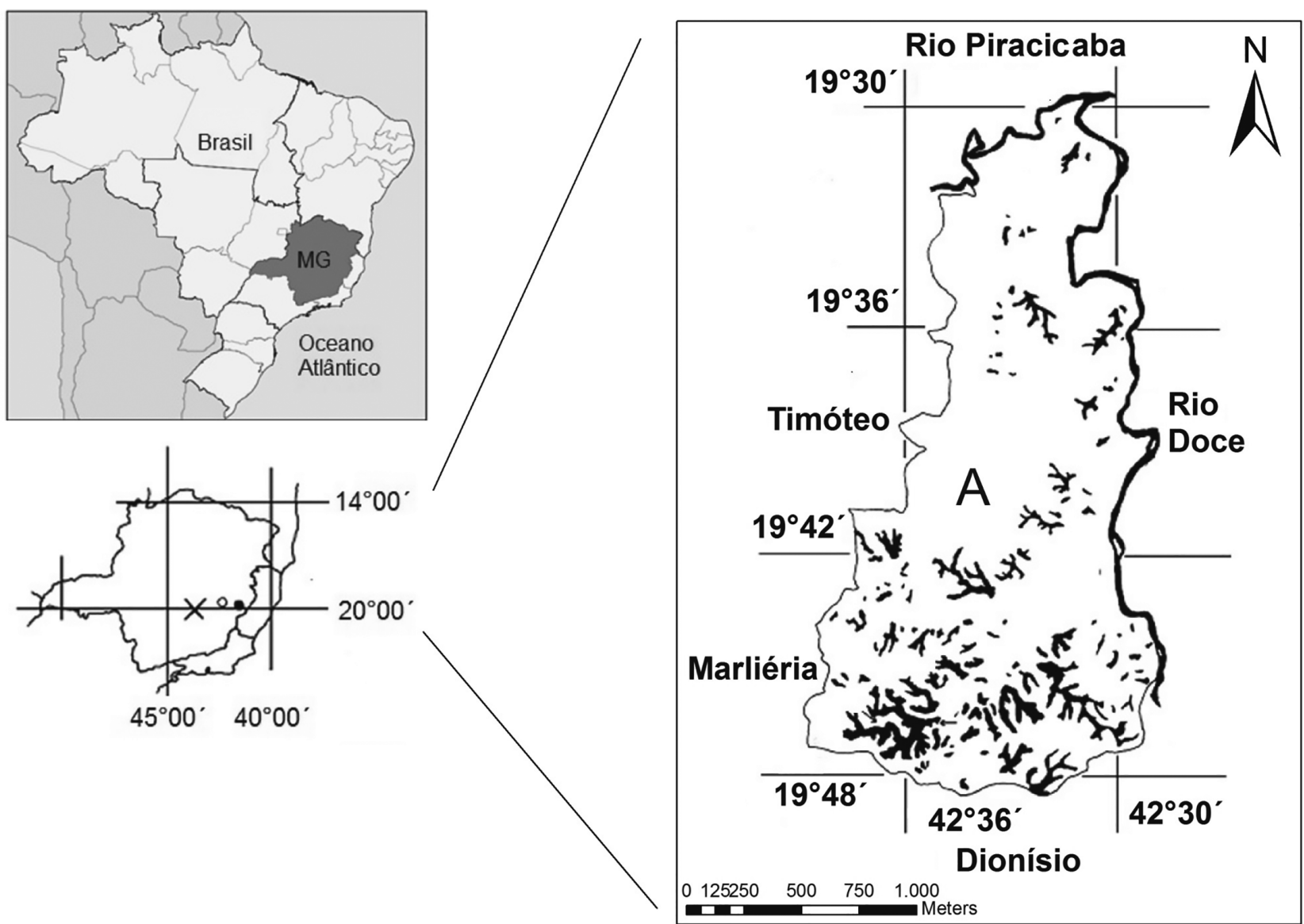

Figura 1. Mapa de localização do PERD. Parque Estadual do Rio Doce, MG, Brasil (modificado de Justo, 2003). A, Lago Lc2.

Figure 1. Location map of the study area. Doce River State Park, Minas Gerais State, Brazil (modified from Justo, 2003). A, Lake Lc2.

com os procedimentos palinológicos não oxidantes descritos por Tyson (1995), Mendonça Filho (1999) e Mendonça Filho et al. (2002), que consistem, basicamente, no tratamento com ácido clorídrico e fluorídrico para eliminação da fração mineral. Para a montagem das lâminas organopalinológicas utilizou-se a fração do sedimento concentrada maior que $2 \mathrm{~mm}$, goma de acácia e Entellan.

\section{Datação radiométrica $\left({ }^{14} \mathrm{C}\right)$}

Para estabelecer a cronologia do testemunho estudado, foram datados pelo método do radiocarbono $\left({ }^{14} \mathrm{C}\right)$ quatro níveis de material carbonoso, sendo elas a $80 \mathrm{~cm}, 65 \mathrm{~cm}, 35$ $\mathrm{cm}$ e $7 \mathrm{~cm}$ de profundidade. Estas amostras foram analisadas pelo Laboratório Beta Analytic Inc $\odot$. (Florida, EUA), que já forneceu as idades calibradas.

As idades pontuais de cada amostra foram obtidas através de interpolação linear e as amostras que estavam fora do range datado, foram obtidas por extrapolação.

\section{Análise granulométrica}

As amostras do testemunho Lc2-01 foram secas, quarteadas, desagregadas e colocadas em sacos plásticos distintos para serem peneiradas. As porcentagens de cada uma das frações granulométricas foram obtidas no laboratório de sedimentologia/ DEGEO/UFOP, pelo método de peneiramento, utilizando a escala de Wentworth (1922). As porções silte/argila foram definidas por serem menores que 0,063 milímetros, porém não houve separação específica das mesmas.

\section{Carbono Orgânico Total (COT)}

As análises de carbono orgânico total foram realizadas no Laboratório de Palinofácies e Fácies Orgânicas da Universidade Federal do Rio de Janeiro (UFRJ). Os valores de COT expressam a quantidade de matéria orgânica contida nos sedimentos (Chagas et al., 2009). Utilizou- se o forno de indução do determinador de carbono WR-12 LECO. A partir de um detector de infravermelho, o analisador SC-144DR LECO quantificou simultaneamente a "concentração" de carbono (COT).

\section{Análise da matéria orgânica particulada (MOP)}

A análise de palinofácies abrangeu a parte qualitativa (descrição das partículas orgânicas) e quantitativa (contagem de 300 partículas) dos grupos e subgrupos de componentes da matéria orgânica particulada, utilizando microscopia sob a luz branca transmitida, nos aumentos de 250 e 400x do microscópio Olympus BX4. A análise em fluorescência dos 
componentes do MOP foi realizada pelo microscópio Zeiss Scope A1 no Laboratório de Paleoecologia Vegetal do Museu Nacional/UFRJ.

\section{Classificação da matéria orgânica particulada (MOP)}

Para a análise de palinofácies, a MOP foi dividida entre os três principais grupos e seus subgrupos: matéria orgânica amorfa (MOA), fitoclastos e palinomorfos.

O Grupo MOA compreende todo componente orgânico particulado sem estrutura, incluindo matéria orgânica amorfa, derivada de bactérias, fitoplâncton, resinas de vegetais superiores e agregados orgânicos degradados. Outros materiais amorfos produtos de vegetais superiores também estão incluídos nesse grande grupo (Mendonça Filho et al., 2010), porém não são considerados MOA no seu sensu usual (Tyson, 1995). A MOA possui a capacidade em formar grumos ou podem ser encontrados finamente dispersos (Chagas et al., 2009). O estado de preservação da MOA é importante na determinação das condições deposicionais do material (Mendonça Filho et al., 2010).

O Grupo Fitoclastos corresponde à fração de tecidos provenientes dos vegetais superiores, sendo divididos em opacos (pretos, carbonizados), não opacos (translúcidos), tecidos cuticulares e hifas de fungo, onde sua classificação e identificação são de suma importância para entender tanto o processo de preservação seletiva, quanto à relação proximaldistal das contribuições de matéria orgânica terrestre em sítios deposicionais (Mendonça Filho et al., 2010).

No Grupo Palinomorfo estão inseridos os Esporomorfos (grãos de esporos de briófitas e pteridofitas/grãos de pólen de Gimnospermas e Angiospermas), microplâncton de parede orgânica (água doce ou marinho) e Zoomorfos (palinoforaminíferos) (Mendonça Filho et al., 2010). Dentre os três grupos da MOP os palinomorfos são os menos abundantes, sendo eles controlados pela extensão da diluição por fitoclastos ou matéria orgânica amorfa (Tyson, 1993).

\section{Parâmetros paleoambientais em palinofácies}

Parâmetros e razões foram utilizados para acessar informações sobre o paleoambiente, e.g. proximidade da área fonte, preservação da matéria orgânica (Tyson, 1993, 1995; Tyson \& Follows, 2000; Carvalho et al., 2006a,b), tendência proximal-distal, história de transporte e os processos de oxidação (Mendonça Filho et al., 2010), bem como a origem biológica dos componentes orgânicos particulados, possibilitando assim, inferências a respeito das condições ambientais nos locais onde houve o processo de sedimentação. Entre eles foi utilizado razão fitoclasto opaco/fitoclasto não opaco (OP/NOP), fitoclasto opaco alongado/fitoclasto opaco equidimensional (Op-la/OP-eq), tamanho das partículas, Fi-COT (obtido com a correlação dos teores de Carbono Orgânico Total com o total de fitoclastos) e o Índice de Preservação de Fitoclastos (IPF).

Para a observação e identificação do MOP sob luz branca transmitida, foi utilizada a descrição dos autores, Tyson (1995), Mendonça Filho (1999) e Mendonça Filho et al.(2002, 2010).

\section{Análises estatísticas}

Após a análise quantitativa dos grupos e subgrupos da MOP do testemunho Lc2-01, foi realizado o tratamento estatístico dos dados. Esses dados foram recalculados para valores percentuais e submetidos à análise de agrupamento (análise cluster) com objetivo de estabelecer grupos e reconhecer a relação entre eles. Para identificar as palinofácies, foi utilizado o coeficiente de correlação 1-Pearson- $r$ / Modo R, que agrupa as variáveis, representada pelos componentes da MOP. As análises de agrupamento pelo modo-Q, as quais utilizaram os programas STATISTICS (1984-2000) e PAST, com o objetivo de realizar a aglomeração por amostras, possibilitando distinguir os intervalos do testemunho estudado (Lc2-01).

\section{RESULTADOS E DISCUSSÃO}

\section{Datação radiométrica $\left({ }^{14} \mathrm{C}\right)$}

Os níveis de material carbonoso do testemunho (Lc201), datados pelo método do radiocarbono $\left({ }^{14} \mathrm{C}\right)$, forneceram as idades já calibradas de $10.191 \mathrm{cal}$. anos AP $(80 \mathrm{~cm}$ de profundidade), $9.640 \mathrm{cal}$. anos AP ( $65 \mathrm{~cm}$ de profundidade), $7.905 \mathrm{cal}$. anos AP (35 cm de profundidade) e $102 \mathrm{cal}$. anos AP $(05 \mathrm{~cm})$ (Tabela 1$)$.

As amostras de 80,0-85,0 cm e $0,0-5,0 \mathrm{~cm}$ de profundidade, que estavam fora da amplitude datada, evidenciaram $10.375 \mathrm{cal}$. anos AP e cerca de 50 anos AP, respectivamente, assumindo idade holocena para esse testemunho.

\section{Sedimentologia do testemunho Lc2-01}

Em função da análise granulométrica, observou-se que de 10.375 a 10.191 cal. anos AP há um pulso de areia muito grossa a areia fina e de 10.191 a 8.194 cal. anos AP há um predomínio de areia grossa a fina, com predomínio de coloração cinza escuro, demonstrando um ambiente de alta energia. A fácies de 8.194 a 7.905 cal. anos AP, apresenta valores semelhantes de grânulos a silte/argila, evidenciando que a contribuição de sedimentos foi de menor magnitude com relação à energia envolvida no sistema. Já de 7.905 a 102 cal. anos AP observa um aumento da fração fina (areia fina a silte/argila), configurando o processo de decantação do material aportado neste ambiente. A ascensão de silte/argila no testemunho Lc2-01da base para o topo, corrobora que o processo deposicional envolveu baixa energia permitindo a decantação das partículas, característica de ambientes lóticos.

\section{MOP do testemunho Lc2-01}

Observa-se na parte basal do testemunho Lc2-01 os valores de Carbono Orgânico Total (COT) são baixos em relação aos valores em direção ao topo do testemunho, tendo uma média de $4,01 \%$, o que demonstrou uma moderada a boa preservação da matéria orgânica.

Verificou-se no testemunho Lc2-01, que entre 10.375 a 8.194 cal. anos AP houve o predomino do Grupo Fitoclastos (20,7\%), entre 9.062 a 8.194 cal. anos AP ressalta as cutículas degradada e não degradada, com percentual de 4,3\% e 7.905 a 102 anos AP prevaleceu o Grupo MOA (44\%) e os Palinomorfos (14,3\%). 
Tabela 1. Intervalos de calibração das idades $\mathrm{C}^{14}$ do testemunho Lc2-01.

Table 1. Calibration intervals for the $\mathrm{C}^{14}$ ages from the Lc2-01 core.

\begin{tabular}{|c|c|c|c|c|}
\hline Localidade & Profundidade $(\mathrm{cm})$ & $\begin{array}{c}\text { Amostra (Código - } \\
\text { Laboratório) }\end{array}$ & $\begin{array}{c}\text { Idade Calibrada } 2 \delta \\
\text { ( } 95 \% \text { probabilidade) }\end{array}$ & Idade Interpolada \\
\hline & 0 & & & 51 \\
\hline & 5 & Beta 28004 & $102.3+0.4 \mathrm{pMC}$ & 102 \\
\hline & 10 & & & 1.403 \\
\hline & 15 & & & 2.703 \\
\hline & 20 & & & 4.004 \\
\hline & 25 & & & 5.304 \\
\hline & 30 & & & 6.605 \\
\hline & 35 & Beta 366683 & $7.840+40 \mathrm{AP}$ & 7.905 \\
\hline & 40 & & & 8.194 \\
\hline & 45 & & & 8.483 \\
\hline & 50 & & & 8.772 \\
\hline & 55 & & & 9.062 \\
\hline & 60 & & & 9.351 \\
\hline & 65 & Beta 365497 & $9.630+40 \mathrm{AP}$ & 9.640 \\
\hline & 70 & & & 9.824 \\
\hline & 75 & & & 10.008 \\
\hline & 80 & Beta 328008 & $10.240+50 \mathrm{AP}$ & 10.191 \\
\hline & 85 & & & 10.375 \\
\hline
\end{tabular}

As cutículas (camada mais externa da epiderme foliar com coloração de amarelo pálido a marrom claro sob a luz branca transmitida e amarela muito intensa sob a fluorescência) tiveram seu pico em 9.351 cal. anos AP chegando a $11 \%$ na amostra e continuaram bem representativas até 8.194 cal. anos AP. Após 7.905 cal. anos AP o percentual de cutículas foi decrescendo até 102 anos AP.

O Grupo Fitoclasto está presente em todas as amostras, sendo mais representativos nas amostras de 10.375 a 7.905 cal. anos AP. Os fitoclastos opacos (alongados e equidimensionais) apresentaram um percentual de $24,4 \%$ nas amostras, chegando a 58,2\% na amostra de 7.905 cal. anos AP. Os outros componentes do grupo, como não opacos bioestruturados (estriado, listrado, bandado e perfurado) ocorreram em 13,8\% das amostras e as membranas (tecido não celular de formato irregular e aparência degradada, com intensa fluorescência) ocorreram em $0,48 \%$ das amostras.

O Grupo MOA, sob a luz branca transmitida, apresentouse em conjugados de coloração castanha a um tom de amarelo escuro, sendo muito representativa nas amostras do topo do testemunho (7.905 - 102 anos AP). Ao longo do testemunho Lc2-01, a MOA apresentou pouca fluorescência, porém constante, que corresponde ao nível 03 na escala de Tyson (1995). Observou-se o início da preservação da matéria orgânica devido à baixa oxigenação a partir de 6.605 cal. anos AP. De acordo com Tyson $(1993,1995)$ a preservação de matéria orgânica está relacionada às áreas onde há condições redutoras, especialmente em áreas de alta produtividade primária. Mendonça Filho et al. (2010) descreveu que elevados percentuais de matéria orgânica é devido as áreas afastadas de atividade flúvio-deltaica intensa (ambientes de baixa energia).
O Grupo Palinomorfo teve uma maior representatividade de 7.905 a 102 cal. anos AP (14,3\%), observando esporos triletes (Cyathaceae) e monoletes (Blechnaceae e Gleicheniaceae), grãos de pólen (Typhaceae, Chloranthaceae, Malpighiaceae Euphorbiaceae, Asteraceae e Fabaceae) e algas (Spirogyra sp.), esses grupos podem ser observados na Figura 2.

\section{Associação de palinofácies}

No Lc2-01 foram identificados três associações de palinofácies reveladas pela análise de agrupamento (Modo-R), em função do maior grau de similaridade dos componentes da matéria orgânica. O dendrograma (Figura 3) mostrou a primeira associação de palinofácies (Pf1) composta somente de cutículas (degradada e não degradada). A segunda associação de palinofácies é composta por fitoclastos não opaco/não bioestruturado (NONbio), não opaco bioestruturado/opacos (NObio), representando os componentes lenhosos. A terceira associação de palinofácies é composta por MOA e palinomorfos.

\section{Interpretação paleoambiental}

Com base na distribuição estratigráfica quantitativa das palinofácies (Figura 4) e análise de agrupamento pelo modo-Q (Figura 5A) foi possível distinguir três fases: Fase 1 (10.375 - 9.351 cal. anos AP), Fase 2 (9.351 - 7.905 cal. anos AP) e Fase 3 (7.905 - 102 cal. anos AP).

Fase 1. (Fase Fluvial 10.375 - 9.351 cal. anos AP). A fase 1 é representada principalmente por derivados de material de fitoclastos da Pf2. Para o topo da fase observa-se um aumento progressivo da Pf2 acompanhado com a diminuição da Pf3, que representa um material típico de ambientes de menor 


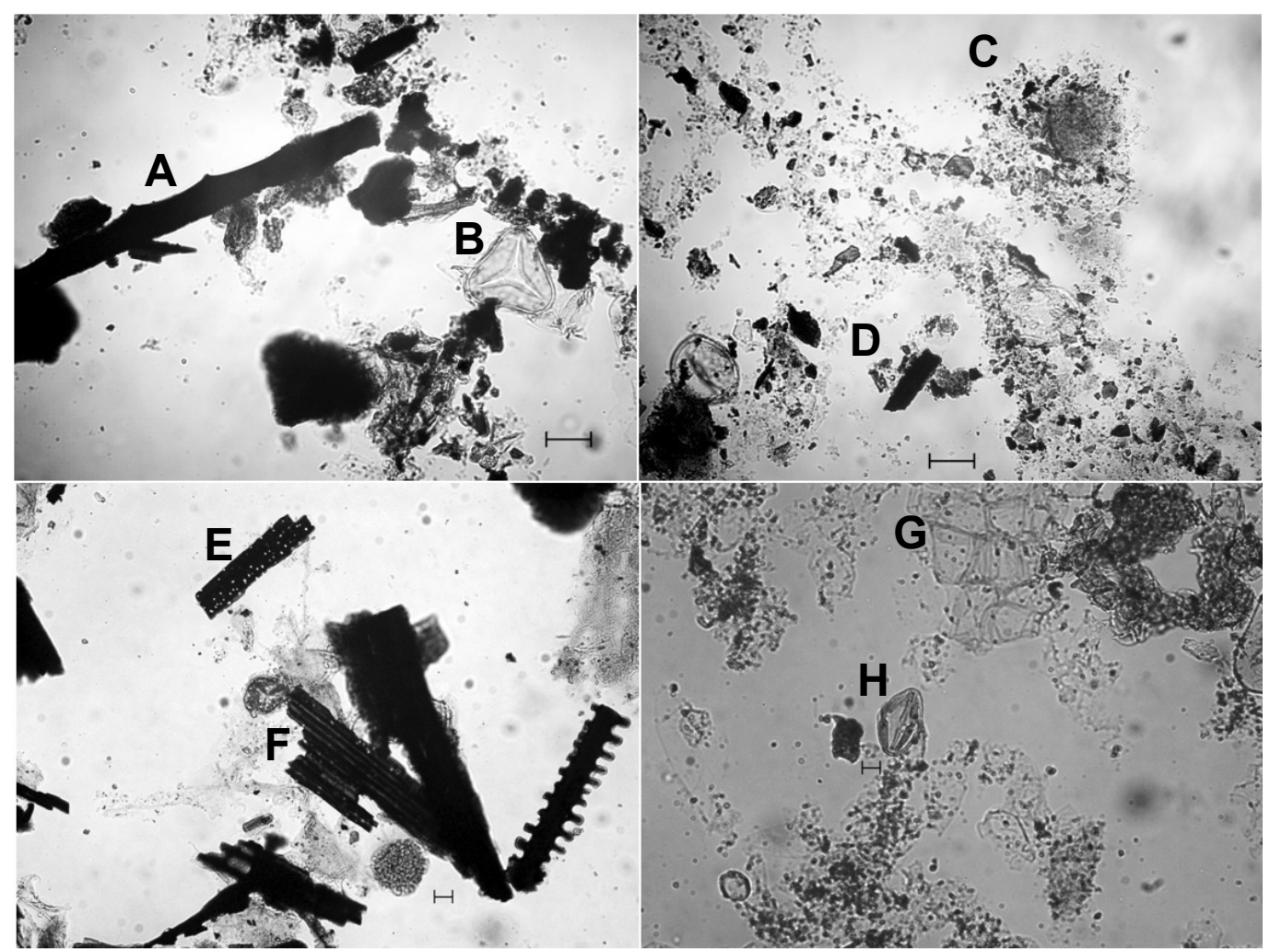

Figura 2. Componentes da matéria orgânica particulada. Abreviações: A, fitoclasto opaco alongado; B, Grão de esporo; C, MOA (matéria orgânica amorfa); D, fitoclasto opaco; E, fitoclasto opaco bioestrururado perfurado; F, fitoclasto opaco bioestrururado estriado; G, cutícula; H, grão de pólen; Escalas $=10 \mu \mathrm{m}$.

Figure 2. Particulate organic matter. Abbreviations: A, opaque lath-shaped phytoclast; B, Spore grain; C, AOM (amorphous organic matter); D, opaque phytoclast; E, bioestructured perforated phytoclast; $\mathbf{F}$, opaque bioestructured striated phytoclast. G, cuticles; H, pollen grain; Scale bars $=10 \mu \mathrm{m}$.

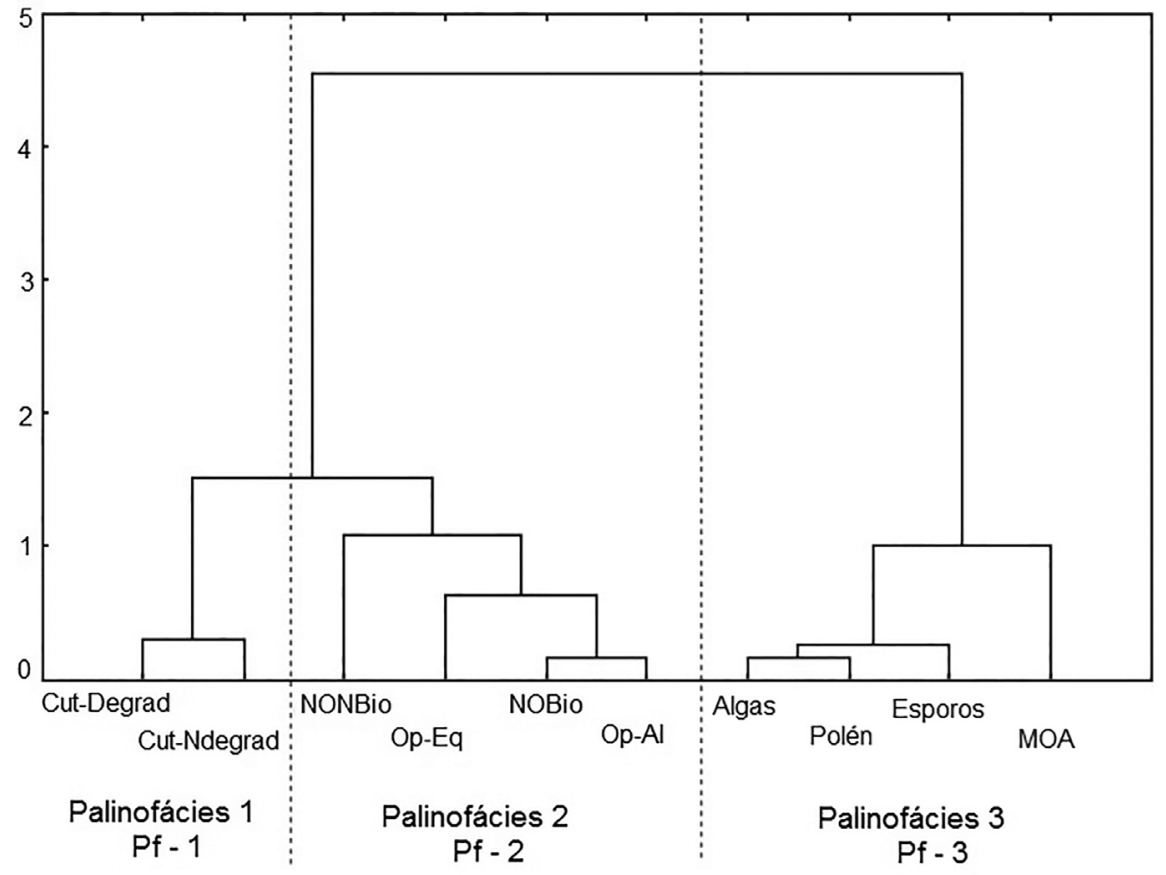

Figura 3. Dendrograma pelo Modo-R do testemunho Lc2-01 revelando as três associações de palinofácies.

Figure 3. Dendrogram by R-Mode of core Lc2-01 showing the three palynofacies. 


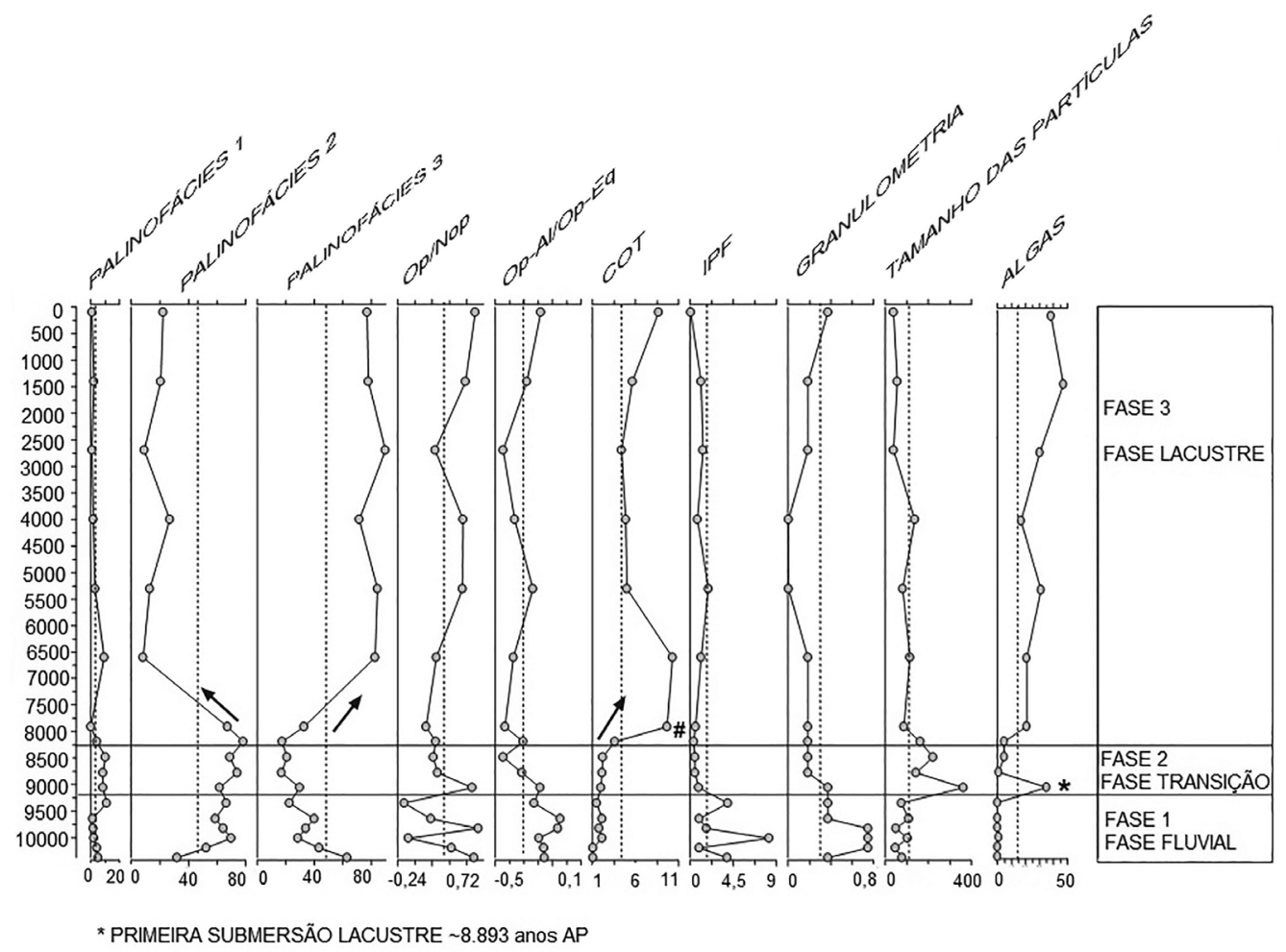

\# ÍNICIO DA MAIOR PRESERVAÇÃO DE MATÉRIA ORGÂNICA DEVIDO A BAIXA OXIGENAÇÃO

Figura 4. Distribuição estratigráfica da matéria orgânica particulada do testemunho Lc2-01. Setas indicam as tendências.

Figure 4. Stratigraphic distribution of particulate organic matter in Lc2-01 core. The arrows indicate trends.

energia (MOA e palinomorfos). Todas as amostras do intervalo, com exceção da primeira amostra (10.375 cal. anos AP, estão acima da média geral da Pf2 (46,8\%). A tendência de aumento de elementos fitoclastos da Pf2 reflete um ambiente com fluxo mais intenso corroborado pela presença de sedimentos de granulometria grossa (acima de $50 \%$ ), baixo teor de COT $(4,01 \%)$ e baixo valor de IPF $(0,43 \%)$. Portanto, as características dessa fase são interpretadas como indicativo de ambiente fluvial que perduraram até cerca de $9.350 \mathrm{cal}$. anos AP. Essa interpretação corrobora os resultados alcançados por Mello (1997), pois de acordo com esse autor, até 9.000 anos $\mathrm{AP}$ a região não tinha sofrido com a movimentação tectônica extensional, responsável pela gênese dos falhamentos que originaram o desnivelamento e basculamento do leito do rio Doce que mais tarde originaram os lagos.

Fase 2. (Fase de Transição 9.351-7.905 cal. anos AP). A fase 2 que compreende o intervalo de 9.351-7.905 cal. anos AP, continua com predomínio da Pf2. No entanto, deve ser destacado que nessa fase observa-se um aumento expressivo da Pfl que é constituída basicamente de cutículas. Em todas as amostras os valores da Pf1 estão acima da média $(4,4 \%)$. O IPF continuou apresentando valores baixos, indicando uma boa preservação dos fitoclastos no ambiente. Em relação à fase 1, nota-se aumento significativo do tamanho das partículas de fitoclastos, sendo registrado o maior pico de tamanho na idade 9.062 cal. anos AP. A granulometria continua sendo grossa, porém abaixo da média $(0,3)$. A razão fitoclastos opacos/ não-opacos (Op/Nop) foi baixa sugerindo que o transporte foi curto e que a deposição ocorreu próximo a área-fonte. No entanto, a abundância das partículas, através da razão opaco alongado/opaco equidimensional (Op-al/Op-eq) demostram também baixo transporte, visto que os opacos equidimensionais são os mais abundantes. A origem das partículas opacas é emblemática, pois não há indicativos de grande transporte e/ou exposição subaérea. Uma possibilidade são os paleoincêndios que podem ter produzido essas partículas. Os baixos valores de COT da fase 01 se mantêm na fase 2. Deve ser destacado que na idade de 9.062 anos AP é registrado um pico de algas. Portanto, são observados em uma mesma idade picos em abundância de algas, tamanho de partícula e da razão Op/Nop. Esses parâmetros conflitantes são típicos de ambiente em transição. Essa ideia corrobora com Overloop (1981) que com base em palinologia, infere mudanças climáticas importantes para mesma faixa de idade, apontando que sucessão de registros de savanas arbustivas frias e secas no Holoceno inicial mudou para condições climáticas e vegetação similar às atuais (Floresta Atlântica Subtropical Úmida). O fechamento dos lagos no PERD é elucidado por estudos geológicos e geomorfológicos que demonstraram movimentos tectônicos que atuaram durante a gênese dos lagos (Barbosa \& Kohler, 1981; Suguio \& Kohler, 1992). Observou também uma significativa diminuição da MOA, coincidente com o aumento de fitoclastos opacos e nãoopacos. Possivelmente, a presença dos fitoclastos de tamanhos 


\section{A}

Distância

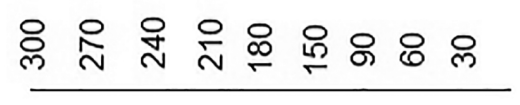

$[102$

$\left[\begin{array}{r}-2703 \\ -203\end{array}\right.$

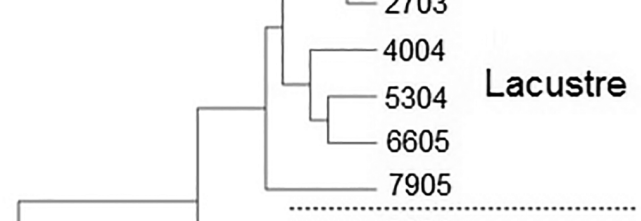

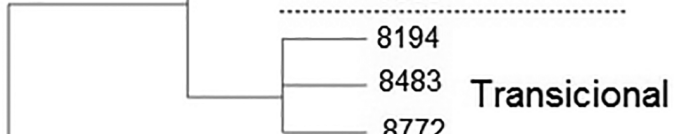
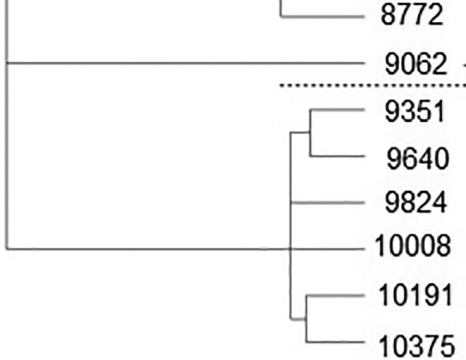

\section{Fluvial}

B

Taxa sedimentação $\mathrm{cm} / \mathrm{anos}$

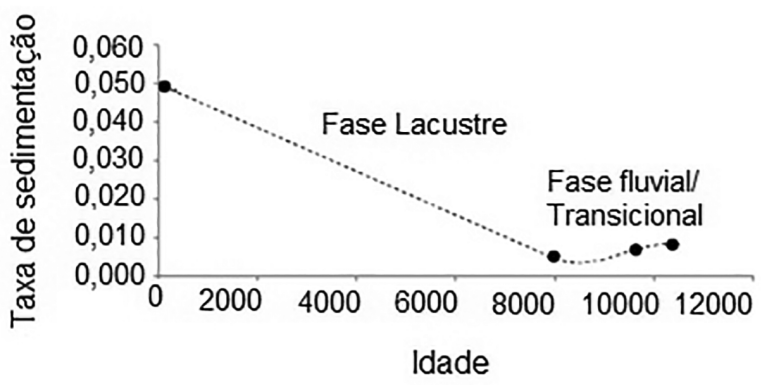

$1^{\circ}$ submersão

Figura 5. A, dendrograma pelo modo-Q do testemunho Lc2-01 revelando as fases deposicionais. B, datação vs. profundidade (cm) mostrando as fases reveladas no dendrograma modo-Q.

Figure 5. A, dendrogram Q-mode of the Lc2-01 showing the depositional phase. B, age vs. depth (cm) showing the depositional phases obtained from dendrogram Q-mode.

avantajados, dispersou a MOA proporcionando a atividade do $\mathrm{O}_{2}$ na coluna d'água e/ou na interface água sedimento.

A primeira submersão lacustre ocorreu por volta de 8.893 cal. anos AP, corroborando com Mello (1997), que realizou análises estratigráficas no Lago Dom Helvécio (PERD) confirmando uma primeira submersão lacustre por volta de 9.000 anos AP, relacionada a lagos, provavelmente rasos e com grande influência de processos aluviais. Os valores homogêneos de granulometria da fácies entre 8.194 a 7.905 cal. anos AP, concomitante com os altos valores de fitoclastos e baixos valores de MOA, sugerem uma fácies proximal flúviodeltaica, podendo ser o inicio do assoreamento do sistema do lago Lc2, sendo a matéria orgânica depositada em ambiente paludal em processo de colmatação. Ybert et al. (1997), afirma que as fácies lacustres foram observadas a partir de 8.500 anos AP o que coincidiu com o desenvolvimento de uma vegetação de floresta semi-decídua. Mello (1997) também afirma que os sedimentos tipicamente lacustres foram identificados somente após 8.200 anos AP. A relação idade vs. profundidade (Figura 5B) mostra que a taxa de sedimentação não foi constante $\mathrm{e}$ que nas fases 1 e 2 a sedimentação foi mais rápida, compatível com ambiente fluvial. Essa interpretação é reforçada pela granulometria mais grossa na base do testemunho.

Fase 3. (Fase Lacustre 7.905-102 cal. anos AP). Na fase 3 , ressalta um aumento expressivo da Pf3, constituída de MOA $(55 \%)$ e palinomorfos $(58,9 \%)$ e uma diminuição brusca da Pf2 e Pf1, refletindo condições redutoras e de baixa energia, demonstrando uma fácies com pouca oxigenação e pouca perturbação da interface água/sedimento de fundo. A granulometria predominante foi de silte/argila, isto é, sedimentos de fração fina. O gráfico apresentado na Figura 5B também reforça essa interpretação, indicado que a taxa de sedimentação não foi constante ao longo do tempo, sendo mais rápido na porção basal (fase fluvial e transicional) e muito lenta na fase lacustre (Fase 3). Esse fato confirma que nessa fase as áreas estavam afastadas de atividades flúvio-deltaicas sugeridas pelos elevados valores de IPF $(1,23 \%)$ e COT $(10,3 \%)$ e principalmente pelos altos valores de MOA. Nota-se nessa fase o início da preservação da matéria orgânica devido à baixa oxigenação por volta de 6.500 anos AP. De acordo com Mello et al. (1999), a idade para os sedimentos do paleocanal do Rio Doce indica que à formação do sistema dos lagos ocorreu após 6.500 anos atrás.

Essa fase corrobora também com o estudo de Silva et al. (2010), onde sugere que o processo de assoreamento da Lagoa Preta (PERD) iniciou provavelmente a partir de 6.620 anos AP. Algas do tipo Spirogyra spp. e grãos de pólen de Typha sp. foram recorrentes e confirmam um ambiente de solos úmidos, pântanos rasos e/ou turfeiras, características típicas de ambiente palustre. Esses indicativos apontam o entulhamento do lago Lc2, o qual se encontra assoreado até à atualidade, sem lâmina d'agua, porém encharcado possibilitando a preservação da MOP. 


\section{CONSIDERAÇÕES FINAIS}

Nos sedimentos recuperados do testemunho Lc201, na área do Parque Estadual do Rio Doce (PERD), a matéria orgânica particulada está associada à ascensão da granulometria fina, evidenciando depósitos do canal fluvial na base, com sedimentos mais grossos, sobrepostos por camadas de areia fina a silte/argila associadas a um ambiente deposicional lacustre holocênico, provavelmente ocasionado por decantação.

Com a caracterização dos tipos de componentes orgânicos particulados e da correlação entre as variações de seus percentuais, foi possível identificar três palinofácies (três fases). A análise de agrupamento modo-R mostrou o grau de similaridade entre os componentes orgânicos, que foram reunidos de acordo com suas tendências de distribuição, evidenciados nas fases 1 a 3, possibilitando assim, fazer inferências sobre as mudanças paleoambientais do lago Lc2 no PERD.

A fase 1 evidenciou que até 9.351 cal. anos AP a região não tinha sofrido com a movimentação tectônica (desnivelamento e basculamento) do leito do rio Doce. A fase 2 (9.351-7.905 cal. anos AP) indicou o possível fechamento do vale do PERD. A fase 3 foi representativa entre 7.905-102 cal. anos AP e sugere a fase lacustre e o inicio do assoreamento do sistema do lago Lc2.

Os resultados obtidos por meio da aplicação de palinofácies, na análise dos sedimentos holocenos do Parque Estadual do Rio Doce, apontaram que este tipo de estudo é uma ferramenta eficaz na interpretação de paleoambientes e na determinação das condições deposicionais da matéria orgânica sedimentar, contribuindo assim, para o entendimento paleoambiental do Quaternário de Minas Gerais.

\section{AGRADECIMENTOS}

O primeiro autor agradece à Coordenação de Aperfeiçoamento de Pessoal de Nível Superior (CAPES), pela concessão da bolsa de Doutorado. Ao Departamento de Geologia da Universidade Federal de Ouro Preto por fornecer a logística necessária à coleta do testemunho e ao desenvolvimento das análises laboratoriais. Aos membros da equipe do Laboratório de Paleoecologia Vegetal/Museu Nacional/Universidade Federal do Rio de Janeiro.

\section{REFERENCIAS}

Barbosa, G.V. \& Kohler, C.H. 1981. O sistema Lagunar do Parque Estadual do Rio Doce, MG. Boletim da Sociedade Brasileira de Geologia, 2:37-46.

Bloom, A.L. 1978. Geomorphology - A systematic analysis of Late Cenozoic landforms. $1^{\text {a }}$ ed., Estados Unidos, Prentice Hall, $510 \mathrm{p}$.

Carvalho, M.A.; Mendonça Filho, J.G. \& Menezes, T.R. 2006a. Paleoenvironmental reconstruction based on palynofacies analysis of the Aptian-Albian succession of the Sergipe Basin, Northeastern Brazil. Marine Micropaleontology, 59:56-81.
Carvalho, M.A.; Mendonça Filho, J.G. \& Menezes, T.R. 2006 b. Palynofacies and sequence stratigraphy of the Aptian-Albian of the Sergipe Basin, Brazil. Sedimentary Geology, 192:57-74.

Chagas, R.B.A.; Mendonça Filho, J.G. \& Mendonça, J.O. 2009. Caracterização Palinofaciológica de uma Sucessão Sedimentar Oligocênica da Formação Tremembé, Bacia de Taubaté. Revista Brasileira de Paleontologia, 12:257-266. doi.org/10.4072/ rbp.2009.3.08

Combaz, A. 1964. Les palynofacies. Revue de Micropaléontologie, 7:205-218

Forsman, J.P. \& Hunt, J.M. 1958. Insoluble organic matter (kerogen) in sedimentary rocks. Acta Geochimica et Cosmochimica, 15:170-182.

Justo, R.L. 2003. Caracterização Palinológica de Depósitos Fluviais Recentes da Região do Médio Vale do Rio Doce (MG). Programa de Pós-Graduação em Geociências, Universidade Federal do Rio de Janeiro, Dissertação de Mestrado, 78p.

Meis, M.R.M. 1978. As unidades morfoestratigráficas neoquaternárias do médio vale do rio Doce. Anais da Academia Brasileira de Ciências, 49:443-459.

Mello, C.L. 1997. Sedimentação e Tectônica Cenozóicas no Médio Vale do Rio Doce (MG, Sudeste do Brasil) e suas Implicações na Evolução de um Sistema de Lagos. Programa de Pós-Graduação em Geologia Sedimentar, Universidade de São Paulo, Tese de Doutorado, 275p.

Mello, C.L.; Metelo, C.M.S.; Suguio, K. \& Kohler, H.C. 1999. Quaternary Sedimentation, Neotectonics and the Evolution of the Doce River Middle Valley lake System (Southeastern Brazil). Revista do Instituto Geológico, 20:29-36.

Mendonça Filho, J.G. 1999. Aplicação de estudos de palinofácies e fácies orgânica em rochas do Paleozóico da Bacia do Paraná, Sul do Brasil. Programa de Pós-Graduação em Geociências, Universidade Federal do Rio Grande do Sul, Tese de Doutorado, 338 p.

Mendonça Filho, J.G.; Carvalho, M.A. \& Menezes, T.R. 2002. Palinofácies. In: T.L. Dutra (org.) Técnicas e procedimentos de trabalho com fósseis e formas modernas comparativas, Unisinos, p. 20-24.

Mendonça Filho, J.G.; Menezes, T.R.; Mendonça, J.O.; Oliveira, A.D.; Carvalho, M.A.; Sant'Anna, A.J. \& Souza, J.T. 2010c. Palinofácies. In: I. Carvalho (ed.) Paleontologia. Interciência, p. 289-317.

Pflug, R. 1969. Quaternary lakes of Eastern Brazil. Photogrammetria, 24:29-35.

Overloop, E. 1981. Post-glacial to Holocene transition in a peatlayer of Lake Jacare (Rio Doce Basin, Brazil). Bulletin de la Société Belge de La Géologie, de Paléontologie et d'Hydrologie, 90:107-119.

Silva, Y.M.P.; Meyer, K.E.B.; Perônico, C. \& Castro, P.T.A. 2010. Palinofácies de uma sequência sedimentar Quaternária da Lagoa Preta, Parque Estadual do rio Doce, MG, Brasil. Revista Brasileira de Paleontologia. 13:49-56. doi.org/10.4072/ rbp.2010.1.06

Suguio, K. \& Kohler, C.H. 1992. Quaternary Barred Lake Systems of the Doce River (Brazil). Anais Academia Brasileira de Ciências, 64:183-191.

Traverse, A. 1994. Sedimentation of Organic Particles. $1^{\text {a }}$ ed. Cambrige University Press, 647 p.

Tyson, R.V. 1993. Palynofacies analysis. In: D.G. Jenkins (ed.) Applied Micropaleontology, Kluwer, Academic Publishers, p.153-191. 
Tyson, R.V. 1995. Sedimentary organic matter: organic facies and palynofacies analysis. London, Chapman \& Hall, 615 p. doi. org/10.1007/978-94-011-0739-6

Tyson, R.V. \& Follows, B. 2000. Palynofacies Prediction of Distance from Sediment Source: A case from the Upper Cretaceous of the Pyrenees. Geology. 28:569-571.

Wentworth, C.K. 1922. A scale of grade and class terms for clastic sediments. Journal of Geology, 30:377-392.
Ybert, J.P.; Albuquerque, A.L.S. \& Turcq B. 1997. Evolução Paleoclimática e Paleoambiental na Região do Vale Médio do Rio Doce (Minas Gerais, Brasil). In: CONGRESSO DA ASSOCIAÇÃO BRASILEIRA DE ESTUDOS DO QUATERNÁRIO, 6, 1997. Resumos expandidos, Curitiba, 31:511-514.

Received in May, 2013; accepted in February, 2015. 